ISSN 1991-8631

Original Paper

http://indexmedicus.afro.who.int

\title{
Gestion intégrée des ressources en eau dans le bassin versant du lac municipal de Dschang : connaissance et usages
}

\author{
Fowe TAZEN ${ }^{1}$, Mathias Fru FONTEH ${ }^{2 *}$ et Harouna KARAMBIRI ${ }^{1}$ \\ ${ }^{1}$ Institut International d'Ingénierie de l'Eau et de l'Environnement (2iE), Centre Commun de Recherche Eau et \\ Climat, Laboratoire Hydrologie et Ressources en Eau, 01 BP 594 Ouagadougou 01, Burkina Faso. \\ ${ }^{2}$ Université de Dschang, Faculté d'Agronomie et des Sciences Agricoles, Département du Génie Rural, BP 447 \\ Dschang, Cameroun. \\ *Auteur correspondant, E-mail : matfonteh@yahoo.com; Tel : +237 77740863
}

\section{RESUME}

Le développement des ressources en eau du sous-bassin du lac municipal de Dschang suscite un intérêt croissant. En prélude à l'élaboration d'une stratégie de gestion durable de l'eau du sous-bassin, un état de lieux a été réalisé et les effets des variations climatiques sur les ressources en eau analysés. Les données climatiques sur la période 1978-2008, les données secondaires des études antérieures et les données des prélèvements en eau effectués par la Camerounaise des Eaux ont été analysées. Ces données ont été complétées par des informations observées sur le terrain et collectées lors des entretiens avec des acteurs clés. Les ressources naturelles en eau de surface ont été estimées à $24 \mathrm{Mm}^{3}$. Seulement $2 \%$ des eaux de surfaces sont prélevées $(0.48$ $\mathrm{Mm}^{3}$ ), tandis que la capacité du seul réservoir dans le bassin est de $0.45 \mathrm{Mm}^{3}$. Il y a un potentiel d'usage pour la production hydroélectrique en aval du réservoir. La qualité microbiologique du lac laisse à désirer pendant certaines périodes de l'année. La pluviométrie montre une tendance à la baisse d'environ $2 \%$ comparée au module interannuel. Les principaux problèmes identifiés sont la faible coordination des acteurs impliqués dans la gestion, l'envasement de la retenue et le manque de données fiables pour une meilleure prise des décisions. Pour une gestion durable, une approche holistique basée sur les principes de la gestion intégrée des ressources en eau devrait être adoptée.

(C) 2013 International Formulae Group. All rights reserved.

Mots clés : Gestion intégrée, connaissance et usages de l'eau, état des lieux et changement climatique.

\section{INTRODUCTION}

La région de l'Ouest du Cameroun est une zone densément peuplée, près de 128,69 $\mathrm{hbts} / \mathrm{km}^{2}$ (INS, 2010), avec un relief accidenté caractéristique des zones montagneuses. Elle est traversée par plusieurs cours d'eau le long desquels sont installés des villages dont le mode de vie des populations reste traditionnel. Le lac municipal de Dschang est une retenue d'eau artificielle localisée au Nord-Est de la ville de Dschang, chef lieu du Département de la Menoua, dans la région de l'Ouest du Cameroun. Le lac couvre une superficie d'environ 18 ha avec un périmètre de 2,34 km (Lacour et Morand, 2006). En amont du lac, les deux affluents qui l'alimentent, drainent un sous-bassin versant très anthropisé. En outre, depuis quelques décennies, le développement 
accéléré de la ville dans ce secteur engendre des activités, sources d'une augmentation très importante de la charge solide du lac et une dégradation croissante du milieu lacustre, compromettant l'usage de ce dernier. C'est dans ce sous-bassin que la plus grande station de traitement d'eau potable de la ville de Dschang est installée.

Compte tenu de l'intérêt socioéconomique et environnemental que suscite ce lac dans cette localité, il devient impératif d'avoir une bonne connaissance des ressources en eau du lac pour permettre de prévenir les éventuelles crises liées à cet or bleu et conduire à un développement durable. C'est dans cette optique que le projet NantesDschang pour la valorisation du patrimoine environnemental et touristique du lac municipal de Dschang a été conçu et mis en œuvre. Ce travail s'appesantira sur l'évaluation des ressources en eau dans le sous-bassin, l'analyse des différents usages actuels et potentiels de l'eau, l'identification des problèmes liés à la connaissance et à l'usage des ressources en eau, l'examen des variations climatiques sur les ressources en eau et la proposition des réseaux susceptibles de les suivre. Cette étude vise donc à attirer l'attention des décideurs politiques et des responsables qui sont en charge de la gestion des ressources en eau du sous-bassin, la nécessité d'implémenter la gestion intégrée des ressources en eau (GIRE) pour une exploitation rationnelle et permettre ainsi d'envisager différentes utilisations et de prévenir les populations des risques sanitaires liés à la fréquentation du lac. La gestion intégrée par bassin ayant pour fondement premier la connaissance que l'on a des ressources en eau en termes de quantité et de qualité, des usages qu'on en fait, cette étude se focalisera sur les aspects connaissance et usages des ressources en eau aussi bien que sur le cadre de gestion.

\section{MATERIEL ET METHODES Site d'étude}

Le sous-bassin versant du lac municipal de Dschang se situe entre $5^{\circ} 25^{\prime}$ et $5^{\circ} 31^{\prime}$ de latitude Nord et entre $10^{\circ} 04^{\prime}$ et $10^{\circ} 08^{\prime}$ de longitude Est et d'altitude variant entre $1615 \mathrm{~m}$ et $1380 \mathrm{~m}$ au niveau de l'exutoire. Le lac municipal se trouve aux confins Sud-Ouest du sous-bassin versant du bassin versant de la Menoua (Figure 1). Ses coordonnées géographiques, au niveau du repère du barrage sont: latitude $5^{\circ} 20^{\prime} \mathrm{N}$ et longitude $10^{\circ} 04^{\prime}$ E (Kenfack, 2000). Le climat de Dschang est de type équatorial de mousson avec une pluviométrie moyenne annuelle de 1790,5 mm. Les maxima de précipitation se situent en août et septembre. Les températures sont très constantes au cours de l'année et varient d'un minimum mensuel de $18,4{ }^{\circ} \mathrm{C}$ en juillet à un maximum de 23,9 ${ }^{\circ} \mathrm{C}$ en février et mars. On y rencontre des sols hydromorphes et ferralitiques (Segalen, 1967). Le substratum est constitué des roches magmatiques et les dépôts superficiels. La végétation dominante est la savane arbustive avec les forêts de galerie.

\section{Evaluation de la quantité et de la qualité des ressources en eau}

Les données utilisées pour estimer le volume d'eau sortant du sous-bassin sont celles collectées par Tekounegning et al., (2010). La technique du jaugeage au flotteur a été utilisée pour déterminer les débits moyens mensuels au niveau du pont à Tsinkop à quelques kilomètres de l'exutoire du sousbassin versant du lac municipal. Pour avoir les débits à l'exutoire du sous-bassin versant du lac, la superficie du sous-bassin versant dont l'exutoire se trouve au pont de Tsinkop et qui intègre le sous-bassin versant du lac a été déterminée par la méthode des trapèzes, et ensuite on a émis l'hypothèse selon laquelle les aménagements et la couverture végétale 
sont semblables au niveau des deux sousbassins, ce qui a permis d'établir une égalité entre les débits spécifiques moyens mensuels sur les deux sous-bassins. Le volume d'eau moyen prélevé annuellement dans le sousbasin pour l'Approvisionnement en Eau Potable (AEP) par la Camerounaise Des Eaux (CDE) a été estimé à partir des relevés journaliers des volumes d'eau brute au niveau des compteurs à l'entrée de la station de traitement dont la moyenne mensuelle est de l'ordre de $40.000 \mathrm{~m}^{3}$ et la valeur actuelle du taux de desserte avoisine $55 \%$. Le volume de la retenue a été estimé en utilisant la profondeur moyenne du lac et la superficie du lac.

Pour mieux apprécier la qualité des ressources en eau du lac, le rapport final des analyses microbiologique, physico-chimique et biologique des eaux du lac municipal de Dschang pendant la période de juillet 2007 à juillet 2008 commandité dans le cadre du projet Nantes-Dschang a été exploité (Fonkou et al., 2008).

\section{Différents usages de l'eau et identification des problèmes}

Les différents usages de l'eau dans le sous-bassin versant du lac se sont identifiés par observation visuelle sur le terrain pour des usages actuels et par entretiens avec les riverains et quelques responsables de l'Université de Dschang pour les usages potentiels. Les problèmes liés à la connaissance et usages des ressources en eau ont été identifiés auprès des différents laboratoires de recherche de l'Université de Dschang susceptibles de mener des travaux sur les ressources naturelles dans le sous-bassin du lac et des organismes nationaux en charge des ressources en eau. L'identification de ces problèmes s'est basée sur les conséquences directement visibles et potentielles d'une mauvaise connaissance des ressources en eau et de ses utilisations.

\section{Identification des variations climatiques}

Pour identifier les variabilités climatiques, la méthode du filtre passe-bas de hanning d'ordre 2 basée sur l'approche du calcul des totaux pluviométriques pondérés qui se fait au moyen des équations recommandées par Tyson et Dyer (1975), présentées par Assani (1999) et des indices pluviométriques annuels suivant la formule proposée par Nicholson (1988), cité par Savané (2001) a été utilisée.

Les totaux pluviométriques pondérés des deux premières années (1), des années intermédiaires (2) et des deux dernières (3) années de la série sont calculés au moyen des expressions suivantes (Lieunang, 2009): $\mathrm{P}_{1}=0.54 \mathrm{X}_{1}+0.46 \mathrm{X}_{2}$ et $\mathrm{P}_{2}=0.25 \mathrm{X}_{1}+$ $0.5 \mathrm{X}_{2}+0.25 \mathrm{X}_{3}$
$\mathrm{P}_{\mathrm{i}}=0.06 \mathrm{X}_{\mathrm{i}-2}+0.25 \mathrm{X}_{\mathrm{i}-1}+0.38 \mathrm{X}_{\mathrm{i}}+0.25 \mathrm{X}_{\mathrm{i}+1}+$ $0.06 \mathrm{X}_{\mathrm{i}+2}$
$\mathrm{P}_{\mathrm{n}-1}=0.25 \mathrm{X}_{\mathrm{n}-2}+0.5 \mathrm{X}_{\mathrm{n}-1}+0.25 \mathrm{X}_{\mathrm{n}}$ et $\mathrm{P}_{\mathrm{n}}=$ $0.54 X_{n}+0.46 X_{n-1}$

Avec $3 \leq \mathrm{i} \leq \mathrm{n}-2$ et $\mathrm{X}_{\mathrm{i}}$ le total pluviométrique observé au cours année d'ordre i.

Le calcul des indices pluviométriques pondérés annuels permet de visualiser d'excédent et de déficit pluviométriques et se fait au moyen de la formule :

$\mathrm{I}_{\mathrm{p}}=\frac{\mathrm{P}_{\mathrm{j}}-\mathrm{P}_{\mathrm{m}}}{\mathrm{S}}$ Avec

$\mathrm{Ip}_{\mathrm{j}}=$ indice pluviométrique pendant l'année $\mathrm{j}$.

$\mathrm{P}_{\mathrm{j}}=$ total pluviométrique pondéré de l'année $\mathrm{j}$.

$\mathrm{P}_{\mathrm{m}}=$ moyenne interannuelle des totaux pluviométriques pondérés.

$\mathrm{S}$ = écart-type de la série des totaux pluviométriques pondérés annuels.

Les données climatiques ont été fournies par la DMN (2009). 


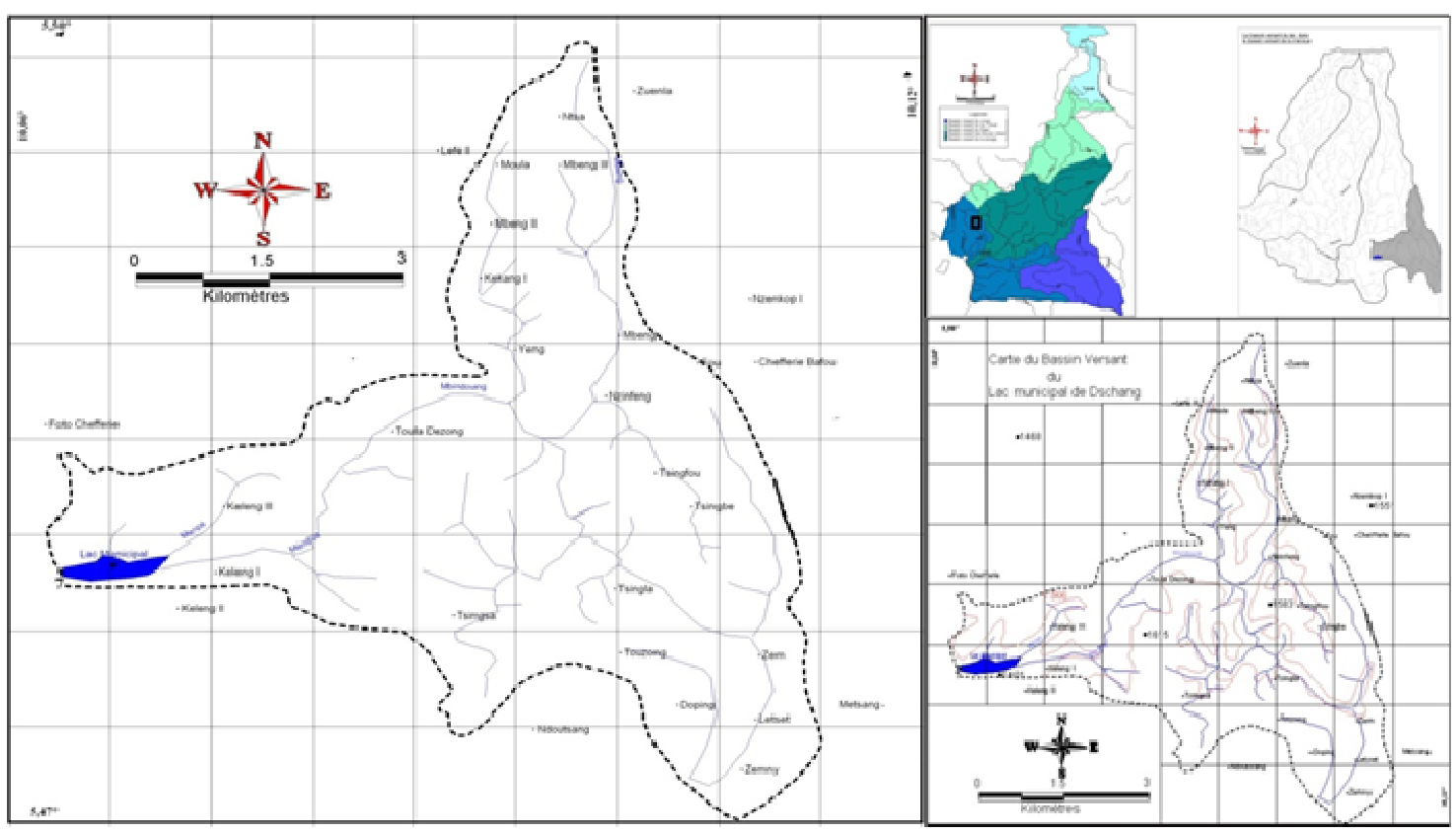

Figure 1: Localisation du lac municipal dans le sous-bassin versant.

\section{RESULTATS}

Evaluation des ressources en eau

Nous avons estimé à 24 millions de $\mathrm{m}^{3}$, le volume annuel d'eau de surface mobilisable dans le sous-bassin versant du lac municipal de Dschang sur la période de 2000 à 2003. Le volume d'eau moyen prélevé annuellement dans le sous-bassin pour l'AEP par la CDE est estimé à 0,48 million de $\mathrm{m}^{3}$ avec une possibilité d'extension à 1,03 million de $\mathrm{m}^{3}$. Le volume de la retenue est estimé à 0,45 millions de $\mathrm{m}^{3}$ (Tableau 1).

Pour ce qui est de l'aspect qualité des eaux, l'analyse bactériologique s'est faite sur les variations en teneurs des paramètres fécaux. Les variations en teneurs des coliformes fécaux et des streptocoques fécaux dans les eaux du lac sont représentées respectivement aux Figures 2 et 3 . Les concentrations des paramètres physicochimiques et des métaux lourds issues des campagnes d'échantillonnage et d'analyse sont regroupées respectivement dans les Tableaux 2 et 3 .

\section{Les usages actuels et potentiels de l'eau}

Une typologie des usages a été faite. Les ressources en eau du bassin du lac municipal sont exploitées pour l'AEP, les activités de loisirs et récréatives, les usages domestiques, les activités agricoles (maraîchage en contre-saison) et potentiellement l'usage hydroélectrique.

\section{Variations climatiques et ressources en eau \\ Les variations des lames précipitées} annuelles (Figure 4) et des indices pluviométriques annuels (Figure 5) ont permis de caractériser les modifications climatiques sur les ressources en eau. Sur la normale considérée, la moyenne interannuelle des précipitations obtenue est de $1790 \mathrm{~mm}$. La gamme de variations des indices pluviométriques se situe dans l'intervalle $8,6 \%$ et $10,7 \%$. Quant aux écoulements, l'hydraulicité moyenne du cours d'eau au pont à Tsinkop est de $0,76 \mathrm{~m}^{3} / \mathrm{s}$. La Figure 6 représente l'évolution des écoulements mensuels dans le sous-bassin sur la période 2000 à 2003.

\section{Problèmes liés à la connaissance et usages des ressources en eau}

Le diagnostic effectué a permis de recenser pas de façon exhaustive les problèmes qui entravent la connaissance et les 
usages des ressources en eau du sous-bassin du lac municipal. Onze problèmes ont été relevés dont quatre liés à la connaissance et sept liés aux usages. Des recommandations sont faites pour le développement des réseaux de suivi hydrologique, hydro-chimique et environnemental des ressources en eau dudit sous-bassin.

\section{Gestion de l'eau dans le sous-bassin}

Depuis l'arrêt du fonctionnement de la centrale hydroélectrique de Dschang dans les années 80, la gestion du lac municipal de Dschang a été concédée à la commune de Dschang sous la responsabilité du magistrat municipal. L'équipe en charge de la commune a entrepris des actions multiples dans le domaine de l'eau, mais ces actions sont malheureusement limitées à cause de la modicité des moyens financiers et techniques dont dispose la commune. Cette étude révèle :

- L'absence d'un cadre de concertation regroupant tous les acteurs (institutionnels, non gouvernementaux et population locale) de l'eau pour débattre des problèmes relatifs aux ressources en eau du sous-bassin et veiller à l'application des lois et règlements sur l'eau en vigueur au Cameroun;

L'absence d'un cadre technique pour évaluer et apprécier les ressources en eau.

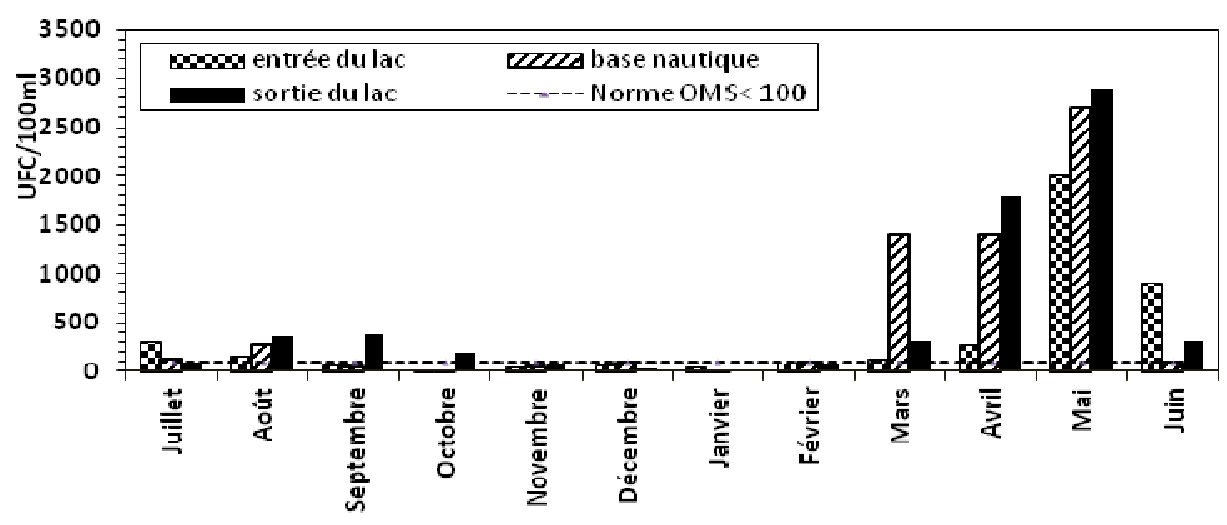

Figure 2: Variation des teneurs en coliformes fécaux dans les eaux du lac municipal de Dschang entre juillet 2007 et juin 2008 .

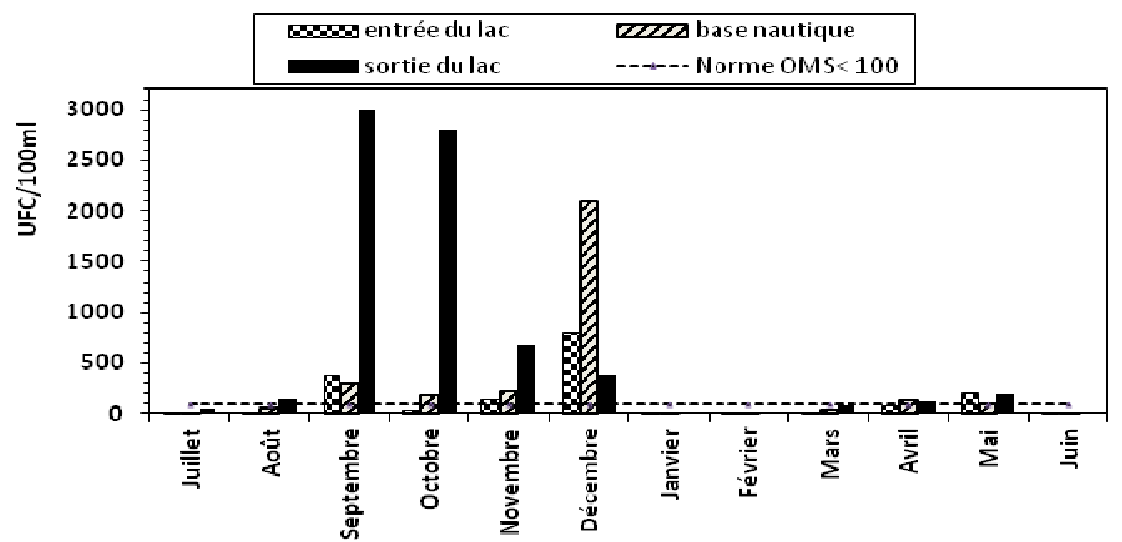

Source: Adaptée de Fonkou et al. (2008) et OMS (2004); N.B: Pour des besoins de lisibilité, celle de septembre a été ramenée à 3000 au lieu de 22000 . 
Figure 3: Variation des teneurs en streptocoques fécaux dans les eaux du lac municipal de Dschang entre juillet 2007 et juin 2008.

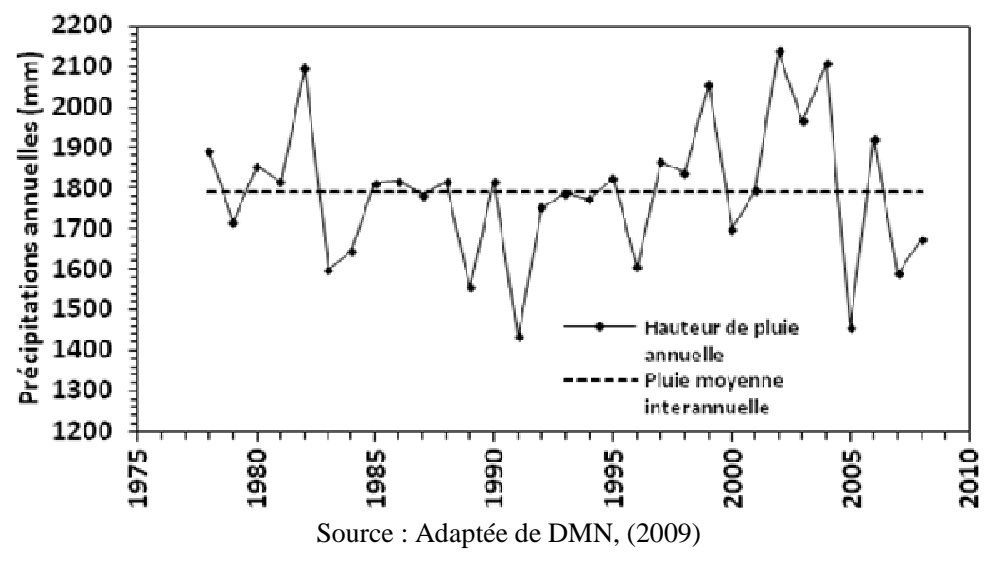

Figure 4: Variation des hauteurs de pluie dans le bassin de 1978 à 2008.

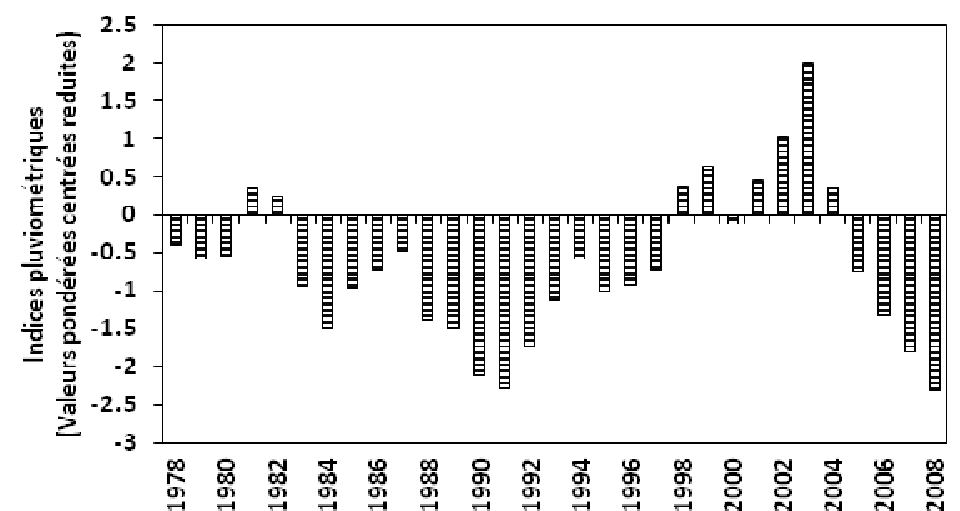

Figure 5: Variation des indices pluviométriques dans le bassin de 1978 à 2008.

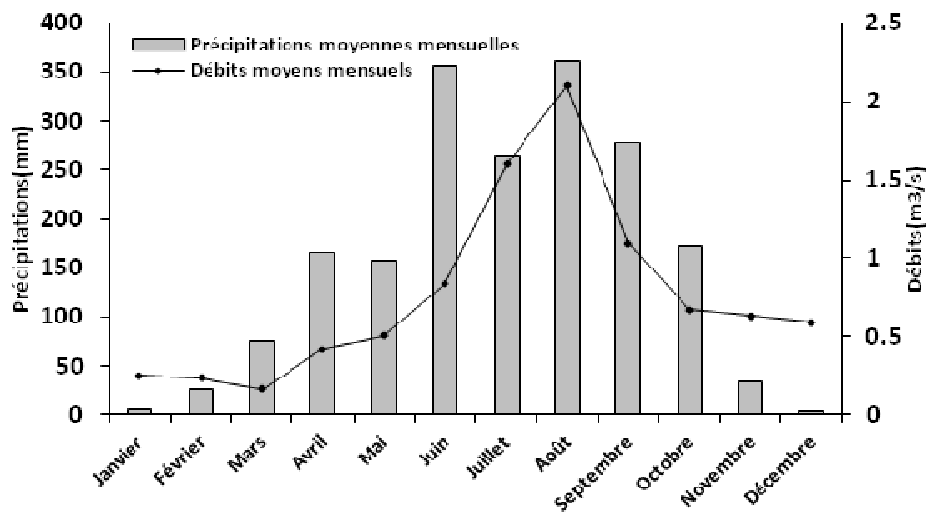

Figure 6: Evolution des débits moyens mensuels en fonction des précipitations moyennes mensuelles de 2000 à 2003. 
Tableau 1: Débits et volumes écoulés moyens mensuels estimés à l'exutoire du bassin versant du lac municipal de Dschang sur la période de 2000 à 2003.

\begin{tabular}{|c|c|c|c|c|c|c|c|c|c|c|c|c|}
\hline Mois & Jan & Fév. & Mars & Avril & Mai & Juin & Juil. & Août & Sept. & Oct. & Nov. & Déc. \\
\hline Débits $\left(\mathrm{m}^{3} / \mathrm{s}\right)$ & 0,252 & 0,235 & 0,168 & 0,420 & 0,505 & 0,841 & 1,598 & 2,102 & 1,093 & 0,673 & 0,631 & 0,589 \\
\hline $\begin{array}{l}\text { Volumes écoulés } \\
\text { mensuels }\left(10^{6} \mathrm{~m}^{3}\right)\end{array}$ & 0,676 & 0,570 & 0,450 & 1,090 & 1,351 & 2,180 & 4,280 & 5,631 & 2,834 & 1,802 & 1,635 & 1,577 \\
\hline
\end{tabular}

Tableau 2: Caractéristiques physico-chimiques des eaux du lac municipal de Dschang pendant les mois de juillet 2007, octobre 2007 et janvier 2008.

\begin{tabular}{|c|c|c|c|c|c|c|}
\hline \multirow{2}{*}{ Paramètres } & \multirow{2}{*}{ Unités } & \multirow{2}{*}{ Directives DCE } & \multirow{2}{*}{ Mois } & \multicolumn{3}{|c|}{ Points de prélèvement } \\
\hline & & & & Entrée du lac & Base nautique & Sortie du lac \\
\hline \multirow{3}{*}{ pH } & & & juil-07 & 7.54 & 6.9 & 6.69 \\
\hline & & $5,5<\mathrm{pH}<9$ & oct- 07 & 6.9 & 6.55 & 6.48 \\
\hline & & & janv-08 & 6.33 & 6.29 & 6.2 \\
\hline \multirow{3}{*}{ Température } & & & juil-07 & 20.8 & 21.7 & 21.6 \\
\hline & ${ }^{\circ} \mathrm{C}$ & 25 & oct- 07 & 21.5 & 21.7 & 21 \\
\hline & & & janv-08 & 22.7 & 22.5 & 22 \\
\hline \multirow{3}{*}{ Conductivité } & & & juil-07 & 41.7 & 69 & 40 \\
\hline & $\mu \mathrm{S} / \mathrm{cm}$ & $<1000$ à $25^{\circ} \mathrm{C}$ & oct-07 & 39 & 41 & 41 \\
\hline & & & janv-08 & 53.6 & 51.4 & 47.2 \\
\hline \multirow{3}{*}{ TDS } & & & juil-07 & 20.8 & 24.8 & 20 \\
\hline & $\mathrm{mg} / \mathrm{l}$ & $<1000$ & oct- 07 & 19 & 21 & 20 \\
\hline & & & janv-08 & 26.7 & 25.8 & 23.5 \\
\hline \multirow{3}{*}{ Turbidité } & & & juil-07 & 27 & 28 & 38 \\
\hline & FTU & $<5$ & oct- 07 & 9 & 12 & 11 \\
\hline & & & janv-08 & 16 & 24 & 13 \\
\hline \multirow{3}{*}{$\mathrm{NO}^{3-}$} & & & juil-07 & 12.3 & 13.3 & 22.1 \\
\hline & $\mathrm{mg} / \mathrm{l}$ & $<50$ & oct-07 & 2 & 0.9 & 1.2 \\
\hline & & & janv-08 & 1.3 & 1.2 & 1.5 \\
\hline \multirow{3}{*}{$\mathrm{NH}^{4+}$} & & & juil-07 & 0.13 & 0.1 & 0.15 \\
\hline & $\mathrm{mg} / \mathrm{l}$ & $<4$ & oct- 07 & 0.14 & 2.14 & 0.45 \\
\hline & & & janv-08 & 0.08 & 0.28 & 0.09 \\
\hline \multirow{3}{*}{$\mathrm{PO}_{3}{ }^{4-}$} & & & juil-07 & 0.16 & 0.11 & 0.33 \\
\hline & $\mathrm{mg} / \mathrm{l}$ & $<5$ & oct- 07 & 0.08 & 0.05 & 0.02 \\
\hline & & & janv-08 & 0.06 & 0.04 & 0.11 \\
\hline \multirow{3}{*}{$\mathrm{SO}_{4}{ }^{2-}$} & & & juil-07 & 0 & 0 & 7 \\
\hline & $\mathrm{mg} / \mathrm{l}$ & $<250$ & oct- 07 & 2 & 2 & 1 \\
\hline & & & janv-08 & 2 & 0 & 1 \\
\hline \multirow{3}{*}{$\mathrm{DBO}_{5}$} & & & juil-07 & 20 & 17 & 14 \\
\hline & $\mathrm{mg} / \mathrm{l}$ & $<5$ & oct- 07 & 15.6 & 16.2 & 13.4 \\
\hline & & & janv-08 & 9.3 & 10.4 & 7.6 \\
\hline \multirow{3}{*}{ DCO } & & & juil-07 & 10 & 9 & 7 \\
\hline & $\mathrm{mg} / \mathrm{l}$ & $<30$ & oct-07 & 9 & 12 & 10 \\
\hline & & & janv-08 & 3 & 10 & 0 \\
\hline
\end{tabular}

Source : Fonkou et al. (2008), DCE (1989) et WHO (2003). 
Tableau 3: Teneurs en métaux lourds des eaux du lac municipal de Dschang pendant les mois de juillet 2007 et janvier 2008.

\begin{tabular}{cccc}
\hline Paramètres & $\begin{array}{c}\text { Directives } \\
\text { DCE, ppm }\end{array}$ & Mois & Entrée du lac \\
\hline \multirow{2}{*}{ Fer } & $<2$ & $\begin{array}{c}\text { juil-07 } \\
\text { janv-08 }\end{array}$ & $\begin{array}{c}1,15 \\
1,45\end{array}$ \\
\hline \multirow{2}{*}{ Cuivre } & $<2$ & $\begin{array}{c}\text { juil-07 } \\
\text { janv-08 }\end{array}$ & $\begin{array}{c}0,13 \\
0,17\end{array}$ \\
\hline \multirow{2}{*}{ Plomb } & \multirow{2}{*}{0,05} & juil-07 & 0,40 \\
& \multirow{2}{*}{ jinc } & janv-08 & 0,39 \\
\hline \multirow{2}{*}{ Cadmium } & $<5$ & juil-07 & 0,8 \\
& \multirow{2}{*}{0,005} & janv-08 & 0,9 \\
\hline
\end{tabular}

Source : Fonkou et al. (2008) et DCE (1989).

\section{DISCUSSION}

L'évaluation quantitative des ressources en eau du bassin a été sousestimée. Les prélèvements pour les besoins agricoles pendant la contre-saison n'ont pas été évalués, aucune estimation des superficies des bas-fonds étant faite.

On a observé des faibles teneurs en coliformes fécaux des eaux du lac pendant la saison sèche (novembre à février); et des valeurs relativement élevées en début de saison pluvieuse avec un pic de 2900 UFC/100 ml en mai. Quant aux streptocoques fécaux, on constate les plus faibles teneurs aux mois de janvier, février et juin; et des valeurs assez élevées en fin de saison pluvieuse avec un pic de 22000 UFC/100 ml en septembre. On observe aussi une forte concentration de ces paramètres fécaux à la sortie du lac, ce qui peut être expliqué par le fait que cette sortie est en contact avec la grande canalisation des eaux torrentielles, et aussi à la forte accumulation des débris divers au niveau de la digue. Les faibles concentrations des indicateurs fécaux pendant la contre-saison se justifient par le fait que les eaux de ruissellement sont limitées et le lac est alimenté par ses affluents naturels. Les concentrations élevées en indicateurs de contamination fécale observées sont dues aux activités auxquelles certains riverains du lac se livrent pendant les grandes pluies, notamment les vidanges des fosses septiques et des latrines mal dimensionnées.

Du point de vue physico-chimique, les eaux du lac municipal de Dschang sont de bonne qualité surtout au mois de janvier selon les Directives des Communautés Européennes (DCE, 1989) relatives aux eaux superficielles, les apports externes étant limités pendant la contre-saison. Les faibles teneurs en azote et en phosphore témoignent de la rareté des eaux torrentielles pendant la saison sèche. Nous avons observé une dissonance par rapport à la réalité des teneurs en $\mathrm{DBO}_{5}$ et en DCO. Les valeurs élevées de la demande biologique en oxygène $\left(\mathrm{DBO}_{5}\right)$, traduisent la présence d'une forte charge polluante en matières biodégradables dans le milieu lacustre. La biodégrabilité de cette charge favorise la prolifération des plantes aquatiques envahissantes contribuant à la dégradation de la qualité de l'eau et limite le développement des espèces aquatiques.

Les faibles concentrations en métaux lourds dans les eaux du lac, témoignent ainsi l'absence des activités industrielles dans cette zone. Nous avons remarqué néanmoins une teneur relativement élevée en plomb et en cadmium, ce qui peut se justifier par les vidanges des batteries d'accumulateurs au plomb dans les eaux du lac et aux émissions 
provenant des huiles de vidange dans les cours d'eau alimentant le lac. Cette concentration élevée en Plomb peut provenir aussi de la dégradation et de l'érosion des sols.

Malgré ces aspects de qualité pas totalement satisfaisante, les ressources en eau $\mathrm{du}$ sous-bassin du lac municipal sont sollicitées pour multiples usages.

- L'approvisionnement en eau potable avec un prélèvement de $40.000 \mathrm{~m}^{3}$ d'eau par mois en moyenne, soit un volume estimé en moyenne de $480.000 \mathrm{~m}^{3} / \mathrm{an}$. La forte croissance de la ville nécessitera une extension des stations de traitement d'eau potable et aura pour conséquence une augmentation des volumes prélevés. Le taux de la desserte de la ville de Dschang avoisine $55 \%$. Pour satisfaire donc la demande en AEP, il faudra augmenter la capacité de la station de traitement de façon à porter les volumes bruts prélevés à plus d'un million de $\mathrm{m}^{3} \mathrm{~d}$ 'eau par an. En saison sèche, le véritable problème qui se pose est le curage au niveau du point de captage et non celui d'insuffisance en eaux brutes;

- Les activités récréatives et de loisirs (natation, sports nautiques). Cet usage ne consomme pas d'eau mais présente des risques sanitaires pour la population qui fréquente le lac;

- Les usages domestiques notamment la lessive, le lavage de la vaisselle voire celui des personnes ou des voitures qui se font tout au long des cours d'eau en aval du point de captage de la station de traitement;

- L'habitat avec la présence des espèces halieutiques (poissons) dans le lac;

- Pendant la contre-saison, ces ressources en eau sont sollicitées pour des besoins agricoles (le maraîchage dans les bas-fonds) et aussi pour des activités de construction des bâtiments par la population locale.

- Il y a une mini-centrale hydroélectrique abandonnée en aval du barrage qui pourrait être réhabilitée pour produire de l'énergie électrique afin de renforcer le réseau national et faire face au délestage dans la ville de Dschang. L'eau du lac municipal de Dschang pourrait donc être utilisée pour réguler les débits au niveau de la centrale. Considérant que seulement $2 \%$ environ des ressources en eau de surface sont actuellement extraites du sous-bassin, des études devraient être menées pour explorer l'utilisation de l'eau dans le sous-bassin pour la production d'électricité, en plus de ses utilisations actuelles.

L'analyse de la série pluviométrique annuelle sur la période 1978-2008 a montré que les hauteurs de pluies varient considérablement d'une année à une autre. Les valeurs extrêmes minimale et maximale sont respectivement observées en 1991 (1433 $\mathrm{mm}$ ) et en 2002 (2137 mm). La tendance des précipitations dans le sous-bassin présente une baisse de 2,05\% par rapport à la moyenne de $1828 \mathrm{~mm}$ obtenue pour la même région par Yakeu (2002) pour 20 ans d'observation. Cette baisse de précipitations influe forcement sur les lames d'eau écoulées dans le sousbassin.

L'évolution de la courbe de variation des indices pluviométriques présente une succession des périodes humides (1981 à 1982, 1998 à 2004) et des périodes sèches (1983 à 1997, 2005 à 2008) dans le sousbassin. Les périodes sèche et humide comportent respectivement des années remarquables : 1991 avec 8,26\% de déficit et 2003 avec 10,70\% d'excédent par rapport à la moyenne de la période de l'étude.

L'évolution des écoulements dans le sous-bassin est presque similaire à celle des hauteurs des précipitations pour la période de juillet à septembre. Les faibles écoulements observés en février et en mars malgré des hauteurs de pluie moyennes sont dus au fait que ce sont les mois les plus chauds et le sol est très sec. Pendant cette période, le taux d'infiltration de l'eau est assez élevé. La hausse des écoulements mensuels pendant les mois de juillet et août correspond à la hausse des précipitations et à une forte humidité du sol limitant les capacités d'infiltration du sol. Les débits en novembre et décembre sont importants parce qu'on se situe à la fin de la saison pluvieuse. Les débits mensuels varient 
en fonction des variations saisonnières de pluie. Nous avons observé un pic au mois d'août caractérisant le régime à deux saisons. La période 2000 à 2003 étant caractérisée comme une période humide, on peut dire d'après l'évolution des précipitations annuelles que les fluctuations climatiques ont contribué à une augmentation des hauteurs de pluie, soit par conséquent un accroissement des volumes d'eau écoulés dans la zone.

Il est important de maîtriser la dynamique des ressources en eau à l'échelle du bassin afin d'améliorer le processus de gestion de l'eau. De nombreux facteurs entravent à la connaissance des ressources en eau. Le diagnostic effectué permet de relever les problèmes suivants :

- Les écoulements au niveau du sous-bassin ne sont pas suivis, de même que les variations du niveau d'eau dans le barrage ;

-L'indisponibilité des données sur les éléments du cycle hydrologique ;

- Le manque de suivi d'indicateurs pertinents favorisant la connaissance socioéconomique des usages de l'eau ;

- La quantité d'eau disponible exploitable dans le sous-bassin n'est pas maîtrisée.

Concernant l'usage de l'eau, les problèmes qui y sont liés sont multivariés. On a :

- La dégradation de la qualité des ressources eau (pollution) ;

-L'occupation illicite des berges pour des fins agricoles, pour l'urbanisation ou comme dépotoirs d'ordures et de déchets divers ;

-L'envasement et l'ensablement entraînant une forte sédimentation du lac qui conduit à la modification du régime des écoulements ;

- La diminution de la population halieutique du lac en quantité et en variétés (Kuaté, 1998 ; Lacour et Morand, 2006) ;

- Les risques sanitaires ;

- Les conflits d'usages de l'eau restent potentiels ;

-Problèmes environnementaux.

D'après nos investigations, les réseaux de suivi des ressources en eau dans le sousbassin versant du lac sont quasi-inexistants. Pour atteindre les objectifs de la maîtrise des ressources en eau à l'échelle du sous-bassin, il serait souhaitable de concevoir et d'optimiser les réseaux de suivi ci-dessous :

$\checkmark$ Réseau de suivi hydrométrique du sousbassin pour la connaissance générale du régime hydrologique. Pour cela Il faudra :

- Disposer des pluviomètres ou des pluviographes de façon à couvrir tout le sous-bassin et faire des relevés journaliers des hauteurs de pluie ;

- Identifier une section stable à l'exutoire du sous-bassin et installer une station synoptique hydrométrique qui permettra d'effectuer les mesures des débits journaliers à différentes saisons ;

- Placer suivant la topographie du barrage, les échelles limnimétriques ou des thalimèdes pour contrôler les variations de la hauteur d'eau dans le barrage ;

- Réaliser au moins tous les deux (02) ans les campagnes de bathymétrie pour contrôler la dégradation spécifique de la cuvette du lac municipal.

$\checkmark$ Renforcement du réseau de suivi climatique en réhabilitant le matériel de la station météorologique de Dschang pour permettre d'avoir les données telles que la température, l'ensoleillement, l'humidité relative et le vent;

$\checkmark$ Réseau de suivi de la qualité physicochimique et bactériologique des ressources en eau. On doit :

- Identifier les sites d'échantillonnage des eaux de surface et souterraines pour effectuer régulièrement les contrôles de la qualité d'eau ;

- Créer un mini laboratoire au niveau de la base nautique de façon à mesurer les paramètres in situ en temps réel ;

$\checkmark$ Mettre en place une police de l'eau pour surveiller le lac de toute forme de pollution et interdire toute activité dans le sous-bassin susceptible de générer une pollution.

De nos jours le site du lac municipal de Dschang est devenu très attractif et touristique avec la présence d'un musée des civilisations, d'une base nautique et de l'Alliance FrancoCamerounaise de Dschang. Pour consolider 
ces actions et les rendre durables et pérennes, il est impératif de penser à une nouvelle approche de gestion de l'eau de ce sousbassin. Cette gestion sera basée sur une approche participative impliquant toutes les parties prenantes avec la décentralisation de la prise de décision au plus bas niveau approprié, d'où la nécessité d'implémenter la GIRE au sein de ce sous-bassin. Cette implémentation se fera par la sensibilisation de la population d'une part, et d'autre part la création d'un Comité Local de l'Eau (CLE) ou d'une Agence de l'Eau (AE) du sous-bassin sous l'autorité du Préfet du département de la Menoua. Cette structure reposera sur 3 cadres: un cadre institutionnel, un cadre juridique et réglementaire et un cadre technique et de recherche.

\section{Conclusion}

Cette étude a permis de faire un état de lieux des ressources en eau du lac municipal de Dschang axé sur l'aspect connaissance et usages. Le volume en eau de surface mobilisable dans le sous-bassin est estimé à 24 millions de $\mathrm{m}^{3} / \mathrm{an}$. Les ressources en eau dans le sous-bassin du lac sont de bonne qualité du point de vue physico-chimique, cependant la qualité bactériologique et parasitologique n'est pas bonne surtout en début et en fin de saison pluvieuse avec des pics observés des indicateurs de contamination fécale, entraînant des risques importants de maladies hydriques et infectieuses. Les sources de pollution des eaux $\mathrm{du}$ lac sont essentiellement d'origine domestique et agricole, aucune activité industrielle n'étant constatée dans le sousbassin. Malgré la dégradation des eaux du lac, la qualité de ces eaux reste encore relativement bonne à des fins de loisir et des activités nautiques. Cependant, ces eaux doivent être prohibées pour toute utilisation comme eaux de baignade et de boisson sans traitement préalable. Les variations climatiques ont eu pour effet une augmentation de la lame d'eau écoulée dans le sous-bassin pour la période de 2000 à 2003.
Les problèmes de connaissance et d'usages liés aux ressources en eau ont été identifiés dans le sous-bassin du lac municipal de Dschang. Ces problèmes constituent une limite à l'évaluation des ressources eau, à la planification des usages et à une bonne gestion de l'eau dans ce sous-bassin.

\section{REMERCIEMENTS}

Nous remercions Professeur Martin Kuete, responsable du Laboratoire de Géomatique, Université de DschangCameroun pour le support informatique mis à notre disposition. Nos remerciements vont également à la Direction de la météorologie Nationale du Cameroun pour les données climatiques dont nous avions bénéficié et à Dr. Tekounegning pour les données de débits spécifiques.

\section{REFERENCES}

Assani AA. 1999. Analyse de la variabilité temporelle des précipitations (19161996) à Lumbashi (Congo-Kinshasa) en relation avec certains indicateurs de la circulation atmosphérique (oscillation australe) et océanique (el niño/ la niña). Bulletin Sécheresse, 10(4): 245-252.

DCE (Directives des Communautés Européennes). 1989. Décret n²94/1989 de l'eau. Qualité des eaux superficielles destinées à la production d'eau potable. UE, Brussels.

DMN (Direction de la Météorologie Nationale). 2009. Banque de données. DMN Douala-Cameroun.

Fonkou T, Noumssi B, Ndouanla J, Lekeufack M. 2008. Analyses microbiologique, physicochimique et biologique des eaux du lac municipal de Dschang pendant la période juillet 2007-juin 2008. Rapport Technique Final, GIE-GRANLAC. Université de Dschang, DschangCameroun.

INS (Institut National de la Statistique). 2010. Annuaire Statistique du Cameroun 2010. INS: Yaoundé-Cameroun. 
Kenfack V. 2000. Contribution à l'étude sédimentologique des apports détritiques dans la retenue d'eau du lac municipal de Dschang: Impact sur l'exploitation touristique de la retenue. Mémoire de Maîtrise, Université de Dschang, Dschang, p. 76.

Kuaté TIR. 1998. Etude hydrochimique des affluents du lac municipal de Dschang. Mémoire de Maîtrise, Université de Dschang, Dschang, p. 46.

Lacour F, Morand F. 2006. Approche sanitaire et environnementale de la mise en valeur touristique du lac municipal de Dschang. Rapport Mission Waterdev. GIEGRANLAC, Dschang, p. 56.

Lieunang LAR. 2009. Préoccupations environnementales affectant la gestion des ressources en eau dans le bassin versant de la Mefou (Centre-Sud, Cameroun) Mémoire de l'Ingénieur Agronome des Eaux Forêts et Chasses, Université de Dschang, Dschang, p. 113.

OMS (Organisation Mondiale de la Santé). 2004. Directives pour la Sécurité des Eaux de Baignade, Eaux Douces et Eaux Côtières (vol. 1). OMS: Genève.

Savané I. 2001. Variabilités climatiques et ressources en eaux souterraines dans la région semi-montagneuse de Man. Bulletin Sécheresse, 12(4): 231-237.

Segalen D. 1967. Les sols et la géomorphologie du Cameroun. Cah. ORSTOM. Série Pédol., 5(2): 137-187.

Tekounegning, Oumarou A, Fomethe A. 2010. Contributions to modernization of the Dschang micro hydro-electric power plant. Global Journal of Engineering Research, 9(1/2): 73-82.

Tyson PD. Dyer TGJ. 1975. Mean annual fluctuations of precipitation in the summer rainfall region of South Africa. S. Afr. Geogra. J., 57: 104-110.

Yakeu S. 2002. Influence des caractéristiques morphostructurales et lithologiques des formations $\mathrm{du}$ bassin versant $\mathrm{du}$ lac municipal de Dschang sur l'action des systèmes d'érosion. Mémoire de Maîtrise, Université de Dschang, Dschang, p. 54.

WHO. 2003. Guidelines for Safe Recreational Water Environments: Coastal and Fresh Waters (vol. 1). WHO: Geneva-Swiss. 